\author{
REVISTA SABERES EDUCATIVOS \\ JUNIO 2018, N 1, 102-116
}

\title{
FORMACIÓN Y EVALUACIÓN DOCENTE BAJO EL ALERO DEL NEOLIBERALISMO: EL CASO CHILENO
}

\author{
Jennifer Brito Pacheco ${ }^{1}$
}

\begin{abstract}
RESUMEN/ ABSTRACT
En las últimas décadas, en Chile, las políticas educativas han estado vinculadas al sistema económico neoliberal, por lo que las racionalidades gerenciales han permeado las dinámicas y relaciones que tienen lugar en las escuelas. De igual manera, la formación de los docentes se ha desarrollado bajo una lógica racionalista que le atribuye roles que no contemplan todas aquellas dimensiones o aspectos necesarios para su desempeño en el aula. Estas situaciones han afectado las dinámicas e interacciones que ocurren en las escuelas $\mathrm{y}$ han ocasionado una desprofesionalización de los docentes. Este artículo tiene como objetivo reflexionar sobre las tensiones que se evidencian en el sistema educativo chileno y en la formación docente bajo el alero del neoliberalismo. Para ello, se selecciona y analiza información de revistas científicas y libros que consideran las contradicciones de la educación bajo los postulados posfordistas y su relación con la formación y la evaluación docente, considerada esta última como principal mecanismo de ascenso en el desarrollo profesional docente. Las conclusiones de este análisis develan la relevancia que tiene la educación como ente emancipador, y la importancia de los aspectos socioemocionales y del paradigma del profesor en la construcción de las reformas educativas, en especial de aquellas directamente vinculadas a los docentes.
\end{abstract}

Palabras claves: neoliberalismo; formación docente; pensamiento del profesor; desprofesionalización docente; evaluación docente.

In recent decades, in Chile, educational policies have been linked to the neoliberal economic system, which is why management rationalities have permeated the dynamics and relationships that take place in schools. Similarly, the training of teachers has been developed under a rationalist logic, attributing to teachers roles that do not include all those dimensions or aspects necessary for their performance in the classroom. These situations have affected the dynamics and interactions that occur in schools and have caused a de-professionalization of teachers. The

\footnotetext{
1 Universidad Austral, Valdivia, Chile; jenniferdianabrito@gmail.com. Becaria Magíster Nacional, Comisión Nacional de Investigación Científica y Tecnológica, Conicyt, año 2018. Folio 22181432.
} 
purpose of this article is to reflect on the tensions that are evident in the Chilean educational system and teacher training under the banner of neoliberalism. For this purpose, information is selected and analyzed from scientific journals and books that consider the contradictions of education under the post-Fordist postulates and their relationship with teacher training and evaluation, considered the latter as the main mechanism of promotion in teacher professional development. The conclusions of this analysis reveal the relevance of education as an emancipating entity, and the importance of socio-emotional aspects and the paradigm of the teacher in the construction of educational reforms, especially those directly linked to teachers.

Keywords: neoliberalism; teacher training; teacher thinking; teacher deprofessionalization; teacher evaluation.

\section{LA EDUCACIÓN EN UNA SOCIEDAD NEOLIBERAL}

En las últimas décadas han ocurrido grandes transformaciones en la sociedad chilena, las cuales han demandado nuevos conocimientos y diversas habilidades (Marcelo y Vaillant, 2013). Han surgido nuevas formas de trabajo caracterizadas por la automatización, la flexibilidad, el autoempleo, las interconexiones y el teletrabajo. Han aparecido renovadas configuraciones familiares, con dinámicas y roles impulsados por la incorporación de la mujer al trabajo y el descenso en las tasas de fecundidad. A lo anterior se suma el aumento de la diversidad étnica, cultural y religiosa, desarrollada por medio de procesos migratorios que se han producido, entre otras razones, por conflictos bélicos, religiosos, territoriales, culturales y económicos (Pérez, 2015).

Desde estos nuevos escenarios, surge la incertidumbre frente a un futuro sustentado, en medio de lo que se percibe como arenas movedizas y estructuras oxidadas, por sistemas políticos, sociales y culturales controlados por una economía neoliberal, en donde el consumismo es el que orienta frente a las bifurcaciones y todos los caminos llegan al mercado.

Estos terrenos pantanosos no han estado alejados del sistema educativo. Es más, se observa que la escuela se ha apropiado de conceptualizaciones y prácticas gerenciales o empresariales, vinculadas a la rendición de cuentas, a estándares, marcos y mediciones que se conjugan para mejorar resultados y alcanzar la tan anhelada calidad. 
En cuanto a los docentes, se hace evidente, de acuerdo a diversas investigaciones (Puga, 2009; Tardif, 2010 y Pérez, 2015) y a los cambios ocurridos en las últimas décadas, que los saberes y conocimientos aprendidos durante su formación de pregrado solo constituyen un cimiento, una base, para iniciar su trayectoria profesional. En este contexto se requiere un nuevo profesionalismo docente en el que se conjuguen formas de aprender a enseñar que se sustenten en la colaboración, en la capacidad de resolver las dificultades de forma colectiva, en la flexibilidad y en la adaptación a los cambios (Hargreaves, 2003). Sin embargo, en la cotidianidad de las aulas, lo que se observa son profesores encarcelados por las demandas y expectativas sociales asociadas al modelo económico reinante. Así, algunos docentes intentan la carrera por el éxito personal y/o evaden enjuiciamientos sobre su labor, convirtiéndose en lo que Hargreaves (2003) ha denominado "víctimas de la sociedad del conocimiento", es decir, promotores del rendimiento, la eficacia, el surgimiento de respuestas esperadas a partir de un marco curricular rígido, parcelado en asignaturas y medido por una evaluación estandarizada que no considera los contextos (Calvo, 2012).

A partir de estas consideraciones, en una primera parte de este artículo, se realiza una reflexión sobre la educación en Chile, para luego analizar las políticas de formación de profesores y la evaluación docente como principal mecanismo de ascenso en el desarrollo profesional a partir de la Ley 20.903 (2016).

\section{CONTRADICCIONES EN LA EDUCACIÓN CHILENA}

Envueltos en el manto gris o blanco del individualismo difundido por los medios de comunicación de masas, habitualmente se escucha a muchas personas expresar sus ideas sobre educación. Una gran cantidad de ellas le asignan un valor de bien de consumo, en donde el objetivo final es la puerta o el puente al éxito económico, a través de los escalones de la movilidad social. Promesa contemporánea democrática de ascenso frente al antiguo sistema de castas o estamentos.

Este juego de intereses creados, pone en evidencia a la educación como algo difuso. Las realidades señalan que en la cancha o en las tablas, la distribución de las oportunidades de experiencias educativas no ha sido equitativa y que se han cometido injusticias garrafales a lo 
largo de la historia o se han perpetuado, por medio de la reproducción social y cultural, modelos de relaciones de poder y simbólicas heredadas a través del capital familiar (Bourdieu y Passeron, 1977).

En este sentido, Brunner (2010) manifiesta que esta diferencia o discrepancia en el capital cultural produce consecuencias en el lenguaje, en el desarrollo cognitivo y en los resultados de aprendizaje de los estudiantes a lo largo de su trayectoria escolar. Bajo estas ideas, la familia sería un actor todopoderoso y las escuelas tendrían que conformarse con intentar simular el proceso de enseñanza-aprendizaje en más de 12 años de escolarización.

Surge así la interrogante: ¿qué aporta la educación a la sociedad? La respuesta podría ser, tal vez, muy poco, pues en una especie de predestinación del dios dinero o dinerocracia, y de supremacía de los aspectos familiares socioeconómicos, los estudiantes pasarían sus días transitando por los establecimientos, como en una romería, a un destino forjado desde el vientre.

Esta situación se puede explicar porque el foco ha estado en la hegemonía de la escolarización, por sobre la educación. Ambos conceptos presentan diferencias fundamentales, ya que "la educación es el proceso de creación de las relaciones posibles, en tanto que la escolarización es el proceso de repetición de relaciones prestablecidas" (Calvo, 2012, p.18. Este último proceso plantea interacciones lineales, de causa-efecto y enajenación de las libertades personales. Mientras que en la educación, y principalmente en la educación informal, se recogen los orígenes socioculturales, los conocimientos situados y los saberes tradicionales de los pueblos, y se considera al estudiante como un ser humano creativo que participa activamente de su proceso educativo por medio de la toma de decisiones (Calvo, 2012).

Desde esta mirada humanista, emancipadora y crítica que reconoce a los sujetos del microsistema como actores protagonistas, Paulo Freire (1985) señala que la educación es transformación, libertad, reflexión, acción, que reconoce, despierta y eleva la conciencia de las personas frente al mundo que los rodea, permitiéndoles cuestionar y analizar las interacciones que ocurren en la complejidad de la sociedad. Se opone, así, a la concepción bancaria de los sistemas educativos, en donde se vacía en el cerebro de los estudiantes pasivos, conocimientos seleccionados de acuerdo a los mandamientos oficiales y a las demandas sociales. 
En este punto resulta esencial observar la conformación histórica antiemocional de la escuela que niega la naturaleza y relevancia de las emociones, generando tensiones, frustraciones, vergüenza y culpa entre sus participantes (Casassus, 2006). Dicho de otra manera, al cumplir con los requerimientos escolares, los sujetos participantes aprenden a actuar de acuerdo a las convenciones sociales, alejándose de sus propias emociones e identidades, a través de una simulación aprendida por ensayo y error, heredada de generaciones pasadas.

Maturana y Nisis (2002) señalan que comprender la relevancia de las emociones en las relaciones sociales permitiría a la educación convertirse en un espacio de transformación y creación que "llevara al estudiante a ser un ser humano responsable y socialmente consciente y que se respeta a sí mismo" (p. 41). Es evidente que las ideas de estos autores rara vez se llevan a la práctica, ni en la escuela ni en las interacciones habituales. Basta con observar unos minutos la propia cotidianidad, representada en los medios de comunicación de masas, en donde se evidencian hechos de violencia, enajenación y deshumanización. Estos hechos se viven diariamente en los centros escolares, dado que allí los descontentos y frustraciones emocionales escapan de forma desbordada, pues las relaciones son normadas desde una lógica racionalista e instrumentalista, olvidando "que el aprendizaje ocurre en una relación” (Casassus, 2006, p.238), en una conexión que vincula a las personas por medio de las emociones.

Esta realidad puede ser entendida, si consideramos que la escuela y el profesorado, sustentados en los actuales cimientos neoliberales, deben preparar a los sujetos para jornadas laborales productivas, junto con resguardar la tradición y, a la vez, hacer que esta no impida potenciar los nuevos avances o progresos de las sociedades (Cataldo, 2010). Para lograrlo, la institución educativa ha asumido una visión “(...) despolitizadora, funcionarial, que utiliza el pensamiento como medio para la generación, producción y acumulación de riquezas, es decir, limitando las potencialidades (...), adiestrando, domesticando, adoctrinando (...)" (Cataldo, 2010, p.24); en otras palabras, reproduciendo modelos prefabricados sobre los procesos de enseñanza-aprendizaje y las dinámicas que ocurren en la escuela.

Se hace necesario, entonces, reflexionar sobre las contradicciones que se producen, especialmente en escenarios como el chileno, cuando, por un lado, los discursos oficiales proclaman políticas amparadas en conceptos como "convivencia escolar", "resolución de conflictos" e "inclusión escolar" y, por otro lado, se anhela y sueña con una calidad educativa 
alejada de las realidades nacionales y de sus propios protagonistas, es decir, distanciada del mundo interior de estudiantes, directivos, funcionarios, familias, comunidades locales y docentes.

\section{EL PARADIGMA DEL PROFESOR Y LAS POLÍTICAS DE FORMACIÓN DOCENTE EN CHILE}

En Chile, a lo largo de su historia, se han realizado diversas modificaciones en las políticas educativas. En ellas, los docentes han pasado de ser considerados personas que contaban con habilidades instrumentales básicas y un poco de cultura general, a técnicos idóneos de la enseñanza, bajo denominaciones como normalistas, funcionarios públicos, empleados municipales o trabajadores en el limbo de lo particular y lo privado.

Cabe señalar que muchas de estas transformaciones no han incorporado al propio docente. Se han subestimando, así, los costos que implica la implementación de las reformas y las complejidades propias de lo nuevo. El proceso de implementación de las innovaciones educacionales es percibido como algo lejano por estos actores educativos, lo que se ha traducido en resquemores, dificultades en el clima laboral, en las estructuras organizativas, en falta de incentivos, comunicación y recursos (Imbernón, 1998 y Vaillant, 2014).

En esta lógica, al analizar las políticas de formación docente en Chile, resulta necesario observar el paradigma del profesor, entendiendo que este actor es un "sujeto estratégico que toma decisiones a partir de sus teorías y creencias antes, durante y después de la interacción con los alumnos" (Martín y Cervi, 2006, p.420). El foco no estará, entonces, en los resultados sino, más bien, en cómo el docente concibe el proceso de enseñanza-aprendizaje y las dinámicas que ocurren en él. Al reconocer que la enseñanza es un fenómeno complejo, incierto, valórico, que no puede ser comprendido desde las visiones reduccionistas de la "racionalidad técnica" (Martín y Cervi, 2006, p.424), se torna posible observar que el profesor construye un conocimiento situado, acorde a su contexto y a las transformaciones que cotidianamente pueden ocurrir en el aula, decidiendo y actuando por medio de la reflexión personal. 
En efecto, Imbernón (1998) menciona que este enfoque vincula las intenciones docentes con sus quehaceres cotidianos, cimentando su formación profesional y la innovación educativa en los juicios, decisiones y actuaciones que realizan en los contextos psicológicos (teorías implícitas, creencias y valores del profesor sobre la enseñanza y el aprendizaje), ecológicos (recursos, circunstancias externas y entornos) y sociales (grupo clase, microsistema e interacciones con otros grupos) en que se desenvuelven.

En esta línea, la profesionalización docente ha sido un tema controversial, ya que se ha desarrollado a partir de las lógicas capitalistas de organización y gestión posfordianas. Estas racionalidades se alejan de la necesidad de desarrollar un trabajo docente contextualizado, en donde se utilizan tanto los procedimientos técnicos como las características personales del profesor. En otras palabras, las modificaciones impulsadas no han considerado la complejidad de la práctica docente en un escenario de rápido y constante desprestigio social, en donde lo que se espera del profesor se aleja de las cotidianidades que este vive en las aulas, instalándose el tedio y el desagrado en el sentido común de los profesores (Tenti,2008).

De ahí la relevancia de considerar el paradigma del profesor en las políticas educativas, evitando correr el riesgo de una formación docente instaurada "como una imposición arbitraria, aleatoria, no verosímil y poco útil” (Imbernón, 1998, p.144), que ocasiona problemas como la desprofesionalización y ausencia de cultura docente por la carencia de oportunidades de toma de decisiones, la falta de autorregulación de su trabajo, la pérdida de posición y prestigio social, conjugada con sistemas educativos burocráticos y materializados en altos niveles de jerarquía, falta de oportunidades de interacción entre pares, sistemas de incentivos y promoción rígidos y jerarquizados, y tareas restrictivas y repetitivas (Imbernón, 1998; Vaillant, 2009; Marcelo y Vaillant, 2013; Basurto, 2014).

En este último punto, tanto el enfoque del pensamiento o paradigma del profesor como los estudios sobre profesionalización docente (Marcelo y Vaillant, 2013) coinciden en señalar la relevancia de la autonomía profesional. En relación al concepto de autonomía es importante tener presente que la autonomía es una noción "polisémica” (Sánchez, 2009, p.13), pues depende de las teorías o de los contextos en que sea definida. Para Foucault, por ejemplo, el fondo cultural y el contexto histórico en el que está inserto el sujeto elimina la autonomía y la noción de libertad "reduciéndola al puro juego de las estrategias del poder" (citado por Sánchez, 2009, p.11), es 
decir, desde este punto de vista, la propia estructura de las sociedades y su organización a través de la política limitan el actuar del ser humano, en este caso de los actores educativos.

Por su parte, Ávalos, Cavada y Pardo (2010) presentan algunas características distintivas de la autonomía docente que permiten potenciarla y delimitarla. Los docentes autónomos serían aquellos que poseen una capacidad reflexiva profunda que les permite pensar y analizar los contenidos que enseñan y las destrezas que poseen para su ejercicio profesional, así como descubrir y usar variadas estrategias pedagógicas para lograr aprendizajes en sus estudiantes.

Cabe destacar que la reciente Ley de Desarrollo Profesional Docente (Ley 20.903, Mineduc, 2016), considera dentro de sus principios tanto la profesionalidad docente como la autonomía profesional, entendiendo a ambas como componentes esenciales para una carrera paulatina, sustentada en la valorización social de los profesores y en la progresión a través del mérito y de la trayectoria docente.

\section{EVALUACIÓN DOCENTE: DEMANDAS EXTERNAS Y COMPROMISOS PERSONALES DE DESARROLLO PROFESIONAL}

Un componente clave para obtener información sobre el ejercicio docente y su relación con los logros de aprendizaje de los estudiantes es la denominada "evaluación docente". Araya y Vargas (2013) consideran que este proceso no solo debe recoger resultados, sino que también debe promover el desarrollo de nuevos saberes, conocimientos y prácticas, que le permitan a los profesores desenvolverse en diversos escenarios educativos, proponiendo sus propias formas de ser evaluados. Según Tenti (2013), se debe pensar el contexto en el que el docente realiza su trabajo y las particularidades propias de la profesión docente, pues muchas de las políticas de evaluación se han centrado en el sistema económico neoliberal, pensando que el profesor

(...) modifica una materia prima (los alumnos, los cuales pueden ser de 'calidad' variable), utiliza una serie de 'medios de producción' (objetivados: tecnologías pedagógicas viejas y nuevas, y subjetivados bajo la forma de 'saber hacer') y 
elabora un producto: el individuo educado. El producto del trabajo del profesor sería el aprendizaje de los alumnos. (Tenti, 2013, p.134)

Para este mismo autor, es necesario estandarizar para evaluar y "toda estandarización supone una decisión arbitraria, una selección y una exclusión de factores y características. Esta operación técnica acarrea siempre un empobrecimiento" (Tenti, 2013, p.138). Este planteamiento, a lo largo de las últimas décadas, ha encontrado resistencias en algunos gremios de profesores (Fardella, 2012). Sin embargo, para Bonifaz (2011), la evaluación docente cumple un rol social, al hacer evidente las fortalezas y debilidades de los profesionales de mayor impacto en las futuras generaciones.

Sobre este tema, Marcelo y Vaillant (2013) señalan que en América Latina hay pocas experiencias sistematizadas de evaluación docente, destacándose en esta materia países como Colombia y Chile. En el primero, en los últimos años, se ha promovido la excelencia profesional y la evaluación, a partir de la cual los profesores son clasificados en escalafones de acuerdo a su desempeño, el que es medido por el cumplimiento de metas señaladas en los planes de desarrollo anuales que los docentes establecen en conjunto con los directores de los centros escolares. Por su parte, en Chile, la evaluación docente es parte de las políticas educacionales desde el año 2003 y se la entiende como un proceso sistemático que busca obtener información sobre el desempeño de los profesores en el aula y el impacto de este desempeño en la calidad del proceso de enseñanza-aprendizaje, con el propósito de fortalecer la profesión docente (Manzi, González y Sun, 2011; Marcelo y Vaillant, 2013; Mineduc, 2016).

Desde su implementación en 2003, se han realizado diversas transformaciones en la aplicación de la evaluación docente siendo la más reciente la del año 2016 con la Ley 20.903, la cual establece que, a partir de este proceso, los docentes serán clasificados en tramos de desarrollo profesional (Manzi, González y Sun, 2011; OECD, 2014; Mineduc, 2016). Precisamente, uno de los asuntos presentes en las negociaciones que han realizado diversos actores (por ejemplo, municipalidades, Colegio de Profesores y Mineduc), al desarrollar los procesos de evaluación docente a lo largo de estos años, ha sido las mejoras en las condiciones laborales y la creación de sistemas de reconocimiento profesional. Esta sería, de acuerdo a Vaillant (2013), una situación extraña en el escenario latinoamericano, pues en la mayoría de los 
países de la región no existen incentivos al desempeño docente, más bien se reconoce la fidelidad con las jerarquías que lideran las escuelas antes que el trabajo realizado en las aulas.

El informe TALIS (OECD, 2013) realizado en Chile menciona que para los profesores la retroalimentación entregada a partir de esta evaluación tiene un impacto positivo, aunque entienden que este proceso obedece a ejercicios administrativos que no se vinculan directamente a sus prácticas y que no se condicen con mejoras en su desarrollo profesional y en sus remuneraciones. Cabe destacar que, de acuerdo a este estudio, un porcentaje importante de profesores percibe en este proceso, una amenaza real a su estabilidad laboral.

En efecto, la evaluación del desempeño profesional docente cobra una gran importancia al considerar que los análisis de las prácticas pedagógicas que realicen tanto los otros actores (pares y evaluación de terceros) como el mismo profesor (autoevaluación) repercuten en la autoeficacia, la autoestima, las creencias que tengan los docentes sobre las acciones que realizan dentro y fuera del aula, la valorización del colectivo docente, el desarrollo profesional, las reflexiones pedagógicas y el reconocimiento o exclusión social a partir de los resultados alcanzados.

Bellei (2007), Fardella (2012) y Vaillant (2014) señalan que las políticas de desarrollo profesional docente chilenas promueven la idea de la docencia como una profesión para los mejores estudiantes secundarios, la calidad medida con pruebas estandarizadas, incentivos económicos para aquellos que logren mejores resultados, es decir, se inscriben en la lógica mercantilista de los premios monetarios por logros de objetivo y la competencia individual. Al respecto, Vaillant (2013) señala que si bien resulta relevante considerar el reconocimiento docente, este debe tener un tinte más social y constituir un sustento fundamental para mejorar la autoestima y la autoeficacia de los docentes, más allá de la entrega de recursos pecuniarios.

Otro elemento que se observa en la profesión docente en la actualidad, tanto en Latinoamérica como en Chile, es la división en áreas de expertos, en las cuales los lenguajes e identidades se visualizan alejados entre sí. Parece necesario construir un conocimiento en común, entendiendo que las estructuras son sistémicas y que los seres vivos (docentes) se construyen a partir de los procesos e interacciones que realicen con los otros componentes del sistema (educativo, en este caso) formado por otros seres vivos (Maturana y Varela, 1995). 


\section{REFLEXIONES FINALES}

Es importante señalar que, en los contextos actuales, la educación y los docentes chilenos se encuentran con leyes, marcos y estándares que siguen preceptos del neoliberalismo, evidenciando que el mercado y sus visiones gerencialistas han entrado de lleno en las relaciones humanas que tienen lugar en la escuela. En esta "liquidez", la incertidumbre y desconfianza hacia el otro nos hace desarrollar relaciones pasajeras y momentáneas (Bauman, 2013). De ahí, la gran cantidad de normas, decretos y ordenanzas que regulan las dinámicas escolares.

En el caso específico de los profesores, culturalmente, se han desarrollado mecanismos de control que siguen lógicas racionalistas e instrumentalistas que, a la larga, han provocado una crisis profesional, al asociarlos con valores y elementos identitarios de tipo funcionarial. Para ello, han debido cumplir con tareas, objetivos, evaluaciones estandarizadas, rindiendo en tiempos y espacios determinados.

Es evidente que estas concepciones no contemplan los aspectos emocionales necesarios en todas las relaciones y en especial en el campo educativo, en donde las complejidades de la humanidad se viven cotidianamente.

Cabe destacar que este fenómeno también ocurre por el desconocimiento de los profesores de su propio mundo interior y por cómo observan en los otros actores (pares, estudiantes, directivos, familias, entre otros) características homogéneas, desde la mirada del aparato burocrático de los centros escolares, más que desde la experiencia vivida a través de la enseñanza y aprendizaje en el aula (Casassus, 2006). Esta situación se vincula, posiblemente, con que los docentes, al haber sido estudiantes, ya tienen una visión preestablecida sobre la docencia.

En este contexto, serán las interpretaciones y reflexiones que realicen los profesores sobre su situación actual y sobre los elementos que hacen única a su profesión, las que les permitirán comprender la relevancia de su propia participación en las transformaciones y cambios de los sistemas educativos. Dicho de otra manera, los profesores deben participar de forma activa en el cuestionamiento, indagación e identificación de las problemáticas vinculadas a su propia formación y desarrollo profesional. Es necesario que ellos mismos develen sus pensamientos, creencias y concepciones sobre el ser y el hacer, a partir del análisis de sus propias prácticas 
pedagógicas y de la cultura escolar de la que son parte, reflexionando sobre los diferentes factores que facilitan o dificultan la construcción de la identidad profesional y potenciando, así, mejoras en su quehacer cotidiano.

De no crearse las condiciones para que los docentes realicen estos procesos reflexivos, se corre el riesgo de generar autómatas programados con una receta del ser profesor, con métodos y técnicas, pero sin la preparación necesaria para la contingencia actual. La vorágine de los avances tecnológicos, comunicacionales e informáticos los sobrepasarían, convirtiéndolos en una especie de computador obsoleto, una máquina de reproducir año a año conocimientos descontextualizados, tornándose, así, imposible que asuman el rol de guías de personas para los tiempos actuales.

\section{REFERENCIAS}

Araya, C. y Vargas, É. (2013). Evaluación docente ¿mecanismo equitativo y confiable o proceso viciado que no cumple con su objetivo? Revista Electrónica Actualidades investigativas en educación, 13(1), 1-31. Recuperado de http://www.redalyc.org/html/447/44725654005/.

Ávalos, B., Cavada, P. y Pardo, M. (2010). La profesión docente: temas y discusiones en la literatura internacional. Estudios pedagógicos, 36(1), 235-263. Recuperado de https://dx.doi.org/10.4067/S0718-07052010000100013.

Bauman, Z. (2013). Sobre la educación en un mundo líquido. Conversaciones con Ricardo Mazzeo. Buenos Aires, Argentina: Paidós.

Basurto, M. (2014). La identidad del docente novel nace en la formación inicial. Revista Intercontinental de Psicología y Educación, 16(1). Recuperado de http://www.redalyc.org/html/802/80230114007/

Bellei, C. (2007). Expansión de la educación privada y mejoramiento de la educación en Chile. Evaluación a partir de la evidencia. Revista Pensamiento Educativo, 40(1), 285-211. 
Bonifaz, R. (2011). Origen de la evaluación docente y su conexión con las políticas públicas en educación. En J. Manzi, R. González \& Y. Sun, La Evaluación Docente en Chile (pp. 1334). Santiago, Chile: MIDE UC.

Bourdieu, P. y Passeron, J-C. (1977). Reproduction. in Education, Society and Culture. Londres, Reino Unido: Sagel.

Brunner, J. (2010). Lenguaje del hogar, capital cultural y escuela. Revista Pensamiento Educativo, $46-47, \quad$ pp. 17-44. Recuperado de http://www.pensamientoeducativo.uc.cl/files/journals/2/articles/459/public/459-1025-1PB.pdf

Calvo, C. (2012). Del mapa al territorio escolar. La Serena, Chile: Ediciones Universidad de la Serena.

Casassus, J. (2006). La educación del ser emocional. Santiago, Chile: Editorial Cuarto Propio.

Cataldo, H. (2010). Funcionariado, política y educación. En H. Cataldo, V. Pankova, S. Ascencio y M. Sobarzo, Los confines de lo educativo ( pp. 13-34). Santiago, Chile: Editorial Arcis.

Fardella, C. (2012). Verdades sobre la docencia, efectos y consecuencias subjetivas de la evaluación docente en Chile. Revista de Psicología, 21(1), 209-227. DOI: 10.5354/07190581.2012 .19996

Freire, P. (1985). Pedagogía del oprimido. México DF, México: Siglo XXI.

Hargreaves, A. (2003). Enseñar en la sociedad del conocimiento. Barcelona, España: Octaedro.

Imbernón, F. (1998). La formación y el desarrollo profesional del profesorado. Hacia una nueva cultura profesional (3era Ed.). Barcelona, España: Editorial Graó.

Manzi, J., González, R. y Sun, Y. (Eds.) (2011). La evaluación docente en Chile. Santiago, Chile: MIDE UC.

Marcelo, C. y Vaillant, D. (2013). Desarrollo profesional docente. ¿Cómo se aprende a enseñar? Madrid, España: Narcea. 
Martín, E. y Cervi, J. (2006). Modelos de formación docente para el cambio de concepciones. En J. Pozo, N. Scheuer, M. Pérez, M. Mateos, E. Martin y M. Cruz. Nuevas formas de pensar la enseñanza y el aprendizaje. Las concepciones de profesores y alumnos (419-433). Barcelona, España: Editorial Grao.

Maturana, H. (1997). Emociones y lenguaje en educación y política. Santiago, Chile: Dolmen Ediciones.

Maturana, H. y Nisis, S. (2002). Transformación en la convivencia. Santiago, Chile: OcéanoDolmen Ediciones.

Maturana H. y Varela F. (1995). De máquinas y seres vivos. Santiago, Chile: Editorial Universitaria.

Mineduc (2016). Ley 20.903. Recuperado de https://www.docentemas.cl/docs/LEY-20903-SistDesarrollo-Profesional-Docente.pdf.

Mineduc (2016). Politica nacional docente. Recuperado de http://www.politicanacionaldocente.cl.

OECD (2014). TALIS 2013 Results: An International Perspective on Teaching and Learning. Recuperado de http://www.keepeek.com/Digital-Asset-Management/oecd/education/guiadel-profesorado-talis-2013_9789264²21932-es\#.WOF_7KNDm8U\#page4

Pérez, A. (2015). ¿Qué docente? ¿Para qué escuela? La formación del pensamiento práctico. La escuela desbordada en la era digital. En R. Hevia (Ed.), Formación continua y desarrollo profesional docente. Ponencias del Seminario Internacional, noviembre, 2013. Santiago, Chile: Organización de los Estados Iberoamericanos para la Educación, la Ciencia y la Cultura.

Puga, R. (2009). Formación continua y un mal entendido fundamental: lecciones de la práctica. En C. Sotomayor y H. Walker (Eds.), Formación continua de profesores ¿cómo desarrollar competencias para el trabajo escolar? (pp.149-178). Santiago, Chile: Editorial Universitaria. 
Sánchez, L. (2009). Una revisión epistemológica de la autonomía. IV encuentro del Centro de Reflexión en Política Internacional. La Plata, Argentina. Recuperado de http://www.memoria.fahce.unlp.edu.ar/trab_eventos/ev.889/ev.889.pdf.

Tardif, M. (2010). Los saberes docentes y su desarrollo profesional. Madrid, España: Narcea.

Tenti, E. (2013). Reflexiones críticas sobre evaluación de los docentes. En J. Díaz (Comp.), Realidades y prospectiva educativa. México D.F., México: SNTE. Recuperado de http://www.snte.org.mx/digital/086_SN_2014.pdf

Vaillant, D. (2009). Políticas de inserción de la docencia en América Latina: La deuda pendiente. Profesorado. Revista de Currículum y Formación del Profesorado, 13(1), 27-41.

Vaillant, D. (2013). Formación inicial del profesorado en América Latina: Dilemas centrales y perspectivas. Revista Española de Educación Comparada, (22), 185-206.

Vaillant, D. (2014). Análisis y reflexiones para pensar el desarrollo profesional docente continuo. Educar, Especial 30 aniversario, 55-66. Recuperado de http://educar.uab.cat/article/view/v50-esp-vaillant/pdf-es. 